\title{
Subpathway-LNCE: Identify dysfunctional subpathways competitively regulated by IncRNAs through integrating IncRNA- mRNA expression profile and pathway topologies
}

\author{
Xinrui Shi ${ }^{1, *}$,Yanjun Xu ${ }^{1, *}$, Chunlong Zhang ${ }^{1}$, Li Feng ${ }^{1}$, Zeguo Sun ${ }^{1}$, Junwei Han ${ }^{1}$, \\ Fei Su${ }^{1}$, Yunpeng Zhang ${ }^{1}$, Chunquan $\mathbf{L i}^{2}$, Xia Li ${ }^{1}$ \\ ${ }^{1}$ College of Bioinformatics Science and Technology, Harbin Medical University, Harbin, 150081, China \\ ${ }^{2}$ Department of Medical Informatics, Daqing Campus, Harbin Medical University, Daqing, 163319, China \\ *These authors contributed equally to this work \\ Correspondence to: Xia Li, email: lixia@hrbmu.edu.cn \\ Chunquan Li, email: Icqbio@163.com \\ Yunpeng Zhang, email: zyp19871208@126.com
}

Keywords: InCRNA, mRNA, cancer, pathway identification, competitive regulation

Received: March 24, $2016 \quad$ Accepted: September 02, $2016 \quad$ Published: September 13, 2016

\section{ABSTRACT}

Recently, studies have reported that long noncoding RNAs (IncRNAs) can act as modulators of mRNAs through competitively binding to microRNAs (miRNAs) and have relevance to tumorigenesis as well as other diseases. Identify IncRNA competitively regulated subpathway not only can gain insight into the initiation and progression of disease, but also help for understanding the functional roles of IncRNAs in the disease context. Here, we present an effective method, Subpathway-LNCE, which was specifically designed to identify IncRNAs competitively regulated functions and the functional roles of these competitive regulation IncRNAs have not be well characterized in diseases. Moreover, the method integrated IncRNA-mRNA expression profile and pathway topologies. Using prostate cancer datasets and LUAD data sets, we confirmed the effectiveness of our method in identifying disease associated dysfunctional subpathway that regulated by IncRNAs. By analyzing kidney renal clear cell carcinoma related IncRNA competitively regulated subpathway network, we show that Subpathway-LNCE can help uncover disease key IncRNAs. Furthermore, we demonstrated that our method is reproducible and robust. Subpathway-LNCE provide a flexible tool to identify IncRNA competitively regulated signal subpathways underlying certain condition, and help to expound the functional roles of IncRNAs in various status. Subpathway-LNCE has been developed as an R package freely available at https://cran.rstudio.com/web/packages/SubpathwayLNCE/.

\section{INTRODUCTION}

In recent years, with the development of next generation sequencing technologies, large scale long non-coding RNA (lncRNA) have been identified $[1,2]$. It has been reported that IncRNA play crucial roles in various key biological processes [3-5], including posttranscriptional regulation [6], tumorigenesis and human disease $[7,8]$. Increasing evidence indicated that lncRNAs can competitively regulate mRNAs expression levels by sharing common miRNA binding sites with mRNAs, which is an important widespread layer of RNA regulation
$[9,10]$. The first confirmation for ceRNA hypothesis in mammalian cells is about PTEN and its pseudogene [11]. PTENP1, which is a PTEN pseudogene and contains many seed matches for PTEN-targeting miRNAs, has been experimentally validated that it can act as a ceRNA for PTEN gene $[11,12]$. The study of Wang et al. suggest that lincRNA-RoR may function as a endogenous miRNA sponge to regulate stemness factors (Oct4, Sox2, and Nanog) and then mediate the ESC maintenance and differentiation processes [13]. It has been demonstrated that $\mathrm{H} 19$ which is an oncogenic genes in multiple cancers, function as a competing endogenous RNA to inhibit miR- 
138 and miR-200a and further led to the de-repression of core genes for mesenchymal cells such as ZEB1/ ZEB2 in colorectal cancer [14]. In addition, the study of Sumazin et al. proposed that the RNA-RNA competing interaction network could regulate oncogenic pathways in glioblastoma [15]. As the RNA competitive interaction can impact important functions in disease, identifying lncRNA competitively regulated pathways is thus not only can gain insight into the underlying mechanism and also help for exploring the functional roles of IncRNAs in disease. However, there are few methods can systematically predict dysfunctional pathways competitively regulated by lncRNAs under disease conditions.

Recently, several useful tools that investigate lncRNA function has been developed [16, 17]. For example, Linc $2 \mathrm{GO}$ annotated lincRNA function based on the competing endogenous RNA hypothesis [16]. LncRNA2Function predicted lncRNA function based on their co-expression protein coding genes across 19 normal tissues [17]. However, currently, the function of lncRNAs underlying certain disease condition have not be well characterized. Additional strategy that explore lncRNA functional roles in the disease context is needed. In addition, Li et al. demonstrate that key local subregions, rather than completely pathways, is more subtly explainable to the etiology of diseases $[18,19]$. It suggest that concentrating more attention on subpathways rather than entire pathways might be more meaningful in identification of disease-relevant pathway and explain the functional roles of lncRNAs in disease.

In this article, we proposed a novel method called Subpathway-LNCE, to identify lncRNAs competitively regulated signal subpathways underlying certain conditon. Firstly, KEGG signal pathways were converted into undirected graphs with genes as nodes and regulated relations as edges. Then, we reconstructed condition-specific lncRNA competitively regulated signal pathways (LRSP) based on matched lncRNAmRNA expression profiles and their shared miRNAs. We mapped interesting lncRNAs and genes into LRSP, then located subpahtways within pathways according to the "lenient distance" similarity method [19]. Finally, the significance of candidate subathways was evaluated by using the Wallenius approximation [20]. In the result section, we firstly applied Supathway-LNCE on prostate data set and LUAD data set to demonstrate the effectiveness of our method. Then we analyzed kidney renal clear cell carcinoma(KIRC) data set to detect kidney cancer key competitively regulated lncRNAs that were biologically meaningful. Meanwhile, our results displayed reproducible by analyzing there independent prostate cancer data sets from different data sources. We also tested robust of our method by randomly disturbing matched expression profiles and LRSP.

\section{RESULTS}

We evaluated Subpathway-LNCE method using prostate cancer data sets and KIRC data set. Firstly, we demonstrated the effectiveness of our method by identifying lncRNAs competitively regulated subpathways for prostate cancer. We then detected KIRC key competitively regulated lncRNAs that were biologically meaningful. Meanwhile, our results displayed reproducible by analyzing there independent prostate cancer data sets from different data sources. We also tested robust of our method by randomly disturbing matched expression profiles and LRSP.

\section{Identifying signal subpathways competitively regulated by LncRNAs for prostate cancer}

We first applied Subpathway-LNCE with SRA data set of prostate cancer to assess the effectiveness [21]. Subpathway-LNCE identified 28 significant lncRNAs competitively regulating subpathways involved 26 complete pathways with FDR $<0.01$ (Supplementary Table S1), of which up to 20 were reported to be associated with cancers, and well reported to be associated with tumor occurrence, development and metastasis (Supplementary Table S1). Furthermore, Subpathway-LNCE located key subregions which were more effective. It is obvious that SubpathwayLNCE can detect LncRNAs competitively regulated pathways that are biologically meaningful.

In further analysis, we focused on three subpathways that competitively regulated by lncRNAs (Figure 1). The first is the most significant subpathway path: 04020_1, which was a subregion of calcium signaling pathway (Figure 1A, Supplementary Table S4). Calcium signaling as an intracellular messenger had been confirmed participate in many biological process, which had closely associated with cancers [22]. We then further explored this subpathway, and found that this subregion was competitively regulated by 8 lncRNAs. Among these lncRNAs, RP11-1398P2.1.1, which was a differential lncRNA, was compititive regulator of PPIF. Apoptosis regulator PPIF, deletion or reduction the expression levels of it could suppress cell proliferation and promote cell migration and invasion [23]. Down regulation of lncRNA MEG3 had been reported closely associated with several cancers, such as lung cancer, gastric cancer, etc [24-26]. Moreover, MEG3 played an important role in the molecular etiology of prostate cancer, which suggested the potential application of MEG3 in prostate cancer therapy [27]. The second significant subpathways was path: 04510_1, an important sub region within focal adhesion pathway (Figure 1B, Supplementary Table S5). In this subpathway, extracellular matrix (ECM) was the protein located in the upstream, which played an important role in 
Step1. Constructing candidate lncRNA-mRNA interaction

a construct candidate LncRNA-mRNA interaction

pridicted lncRNA-associated competing triplets

Candidate LncRNA-mRNA interaction
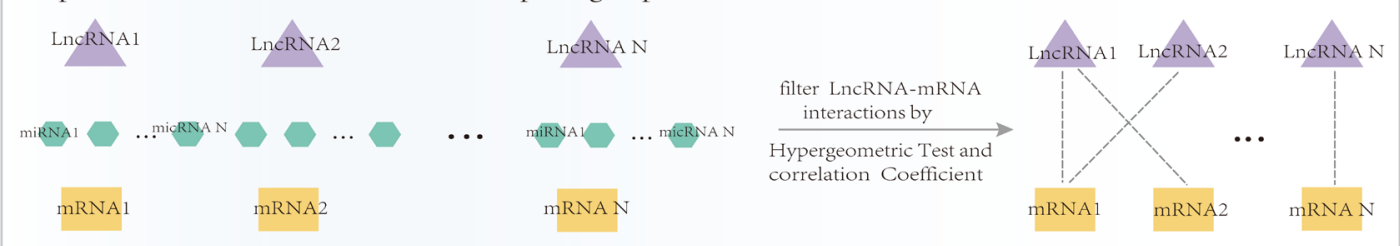

Step2. Link lncRNA to targets whithin pathway graphs

convert all signal pathwys from KEGG Database to undirected graphs with gene as nodes

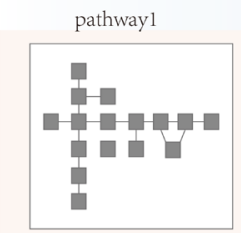

LncRNA profile

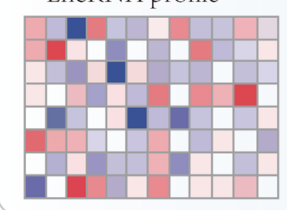

pathway2

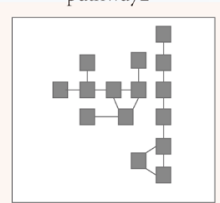

mRNA profile

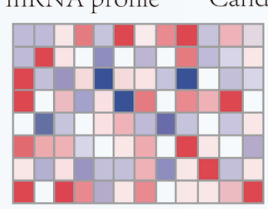

pathway $\mathrm{N}$

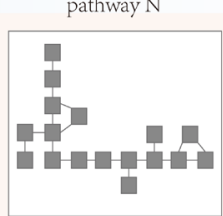

te LncRNA-mRNA interaction

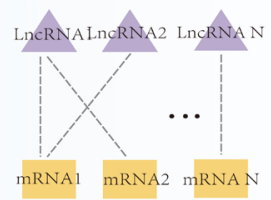

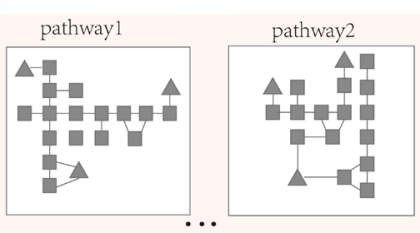

pathway $\mathrm{N}$

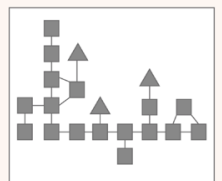

Step3. idetification of lncRNA-regulated subpathways

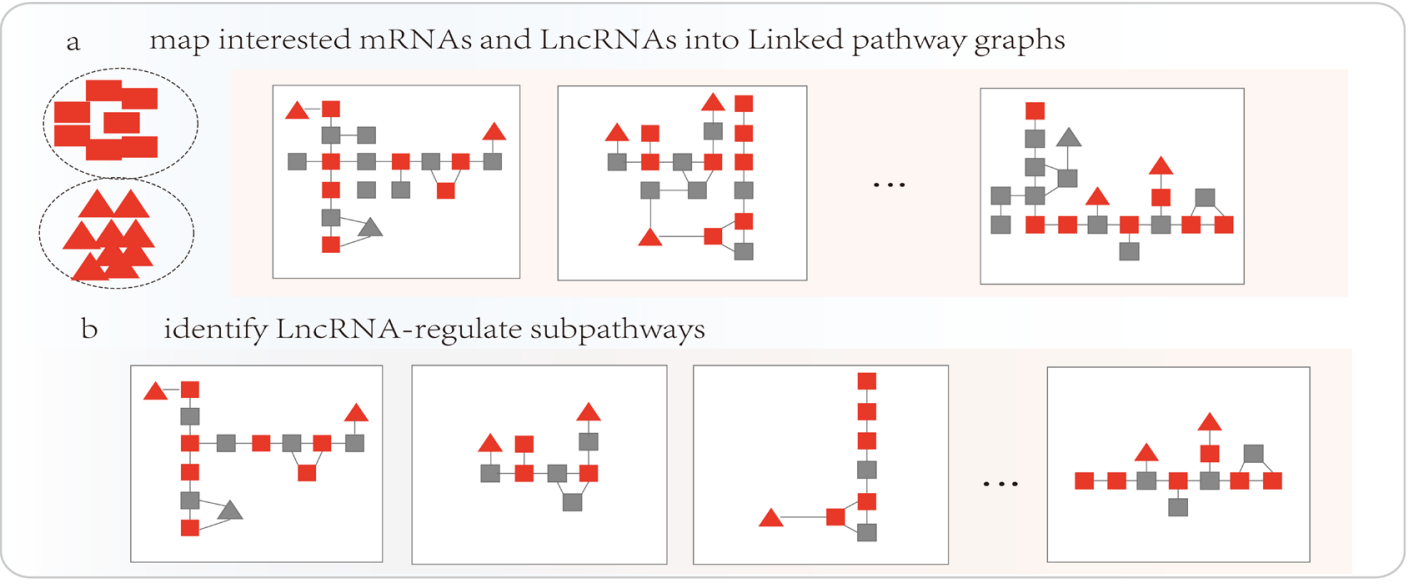

Step4. Test the statistical significance of identified subpathways

Test the statistical significance of identified subpahtways

\begin{tabular}{|c|llll|}
\hline subpathwaylD & pathwayName & AnnRatio & Weight & Pvalue \\
\hline subpathway1 & pathwayName1 & $3 / 56$ & weight1 & pvalue1 \\
subpathway2 & pathwayName2 & $5 / 56$ & weight2 & pvalue2 \\
$\ldots$ & $\ldots$ & $\ldots$ & $\ldots$ & $\ldots$ \\
subpathwayN & pathwayNameN & $4 / 56$ & weightN & pvalueN \\
\hline
\end{tabular}

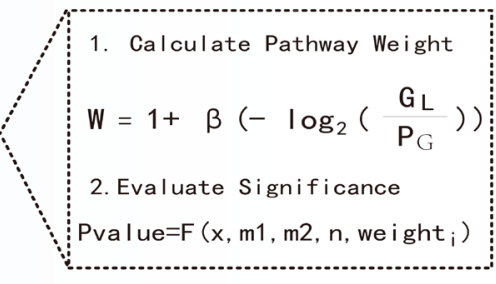

Figure 1: The pipeline overview of Subpathway-LNCE. 
different metastatic contexts in cancers [28, 29]. Notably, it was coordinately regulated by four lncRNAs, MIAT, MEG8, AC005682.5.1, and AC078937.4.1. Among these lncRNAs, MEG8 and AC078937.4.1 were differentially expressed. Another significant differently expressed IncRNA was LINC00087, which competitively regulated vascular endothelial growth factor (VEGF) and cyclin D2 (CCND2). Overexpression of the differential gene VEGF could promote angiogenesis and tumourogenesis in prostate cancer, and targeting the VEGF receptor pathway had shown promising early clinical application $[30,31]$. Although CCND2 was a non-differential gene, LINC00087 which competitively regulated it were differentially expressed. CCND2 had an inhibitory potential on the proliferation of androgen receptor (AR)dependent prostate cancer cells [32]. A high Cyclin D2 methylation levels was related with clinicopathologic features of tumor aggressiveness in prostate cancer [33]. LINC00087 that competitively regulated VEGF and CCND2 suggested that it may play an important role in prostate cancer. Additional, DLEU2 and HOTAIRM1 were involved in this subpathway. These two lncRNAs had been reported play important role in the leukemia cells [3436]. In addition, DLEU2 was the host gene of miR-15a and miR-16-1 which were important tumor suppressors [37]. DLEU2 and HOTAIRM1 competitively regulated PPP1CC and ROCK1 respectively. It was notable that downstream of PPP1CC and ROCK1 was MYL9, which was a famous hallmark tumor gene [38]. Moreover, it had been reported that MYL9 may efficiently predict recurrence-free survivals in prostate cancer patients [39]. In summary, these result suggest that our method can not only identify biological meaningful subpathways, but also highlight some critical lncRNAs underlying disease condition.

The third subpathway, path: 04070_1, was a part of Phosphatidylinositol signaling system (Figure 1C, Supplementary Table S6). In this subpathway, phosphatase and tensin homologue deleted on chromosome-10 (PTEN) was a tumor suppressor gene that was competitively regulated by three LncRNAs, FDG5-AS1, WDFY3AS2 and CTD -2302E22.2.1. PTEN inactivation had a correlation with many different types of cancer including prostate cancer $[40,41]$. Moreover, PTEN negatively regulated activity of the $\mathrm{PI} 3 \mathrm{~K} / \mathrm{Akt} / \mathrm{mTOR}$ pathway, which played a prominent role in prostate tumor development $[42,43]$. We then further focused on the underlying miRNAs that shared by PTEN and these three lncRNAs. We found that most miRNAs were associated with tumor. For example, among them miR-214 was shared by PTEN and all three lncRNAs of competitive regulators, was reported to be diagnostic potential biomarker in prostate cancer urine specimens with qRT-PCR experiment [44]. Mir-17 family were known as oncomiRs and had essential functions for tumorigenesis [45].
In particular, by targeting miR-17-5p and miR-106a-5p, PTEN expreesion were rescued resulting in reducing tumor growth in vivo of prostate cancer [45]. Though functions of FDG5-AS1, WDFY3-AS2 and CTD -2302E22.2.1 were unclear, Subpathway-LNCE reveals that they may play important role in prostate cancer by competing cancer related miRNAs with PTEN to disturb the phosphatidylinositol signaling system.

\section{Identifying signal subpathways competitively regulated by LncRNAs for LUAD dataset}

To examine the utility of Subpathway-LNCE for diseases, we applied Subpathway-LNCE with TCGA data set of LUAD. Subpathway-LNCE identified 28 significant lncRNAs competitively regulated subpathways in LUAD data sets with FDR $<0.01$ (Supplementary Table S3), of which up to 15 were well reported to be associated with tumor occurrence, development and metastasis (Supplementary Table S3). It was note that Small cell lung cancer was on the top rank in the Supathway-LNCE result list. In this subregion, HCG-18 competitively regulated gene CCND1, which was an oncogene acted as a driver of multiple types of human malignancies [46, 47]. CCND1 dysregulation was associated with cellular proliferation and tumor growth of lung tumor [48]. Furthermore, Subpathway-LNCE located key subregions which were more effective. It is obvious that Subpathway-LNCE can detect lncRNAs competitively regulated pathways that are biologically meaningful.

\section{Dissecting key lncRNAs in KIRC- relevant IncRNA competitively regulated signal subpathways network}

In this section, we focused on applying our method to dissect key lncRNAs that implicated with disease and further explore its functional roles under disease condition. To do this, we performed Subpathway-LNCE on KIRC dataset. Firstly, we constructed the KIRC-relevant lncRNA competitively regulated signal subpathways network, in which the top rank 20 subpathways in SupathwayLNCE result list and IncRNA that competitively regulated these subpathways were considered. These 20 subpathways belonging to 19 complete pathways of which up to 14 had been well reported associated with cancers (Supplementary Table S2). Hub nodes are always very important in the biological network as the connectivities of which are extremely high. We thus focused on hub IncRNAs in the IncRNA competitively regulated signal subpathways network. We selected the top $10 \%$ of lncRNAs with the highest degrees in the lncRNAsubpathway network as the hub lncRNAs. We then merging 20 subpathways including relevant mRNAs and lncRNAs to construct KIRC relevant modulatory network. Finally, we obtained the KIRC relevant modulatory 
network involved 19 pathways, 14 hub LncRNAs and 48 gene/proteins (Figure 2A).

The modulatory relationship HCG18- EGFR appeared up to 10 pathways, of which 7 pathways were cancer-related such as focal adhesion, such as MAPK signaling pathway and etc. EGFR was a receptor tyrosine kinase, its signaling ultimately increased angiogenesis and decreases apoptosis [45]. Overexpressed gene EGFR was a famous driver gene in mass of human tumors and had well be used as targeted therapy $[49,50]$. Furthermore, it had experiments demonstrated dysregulation of the EGFR pathway was associated with growth and invasion in cancer [51]. HCG-18 competitively regulated another gene CCND1, which was an oncogene acted as a driver of multiple types of human malignancies [46, 47]. CCND1 dysregulation was associated with cellular proliferation and tumor growth of kidney tumor [52]. Another differential hub lncRNA PVT1 was also competitively regulated CCND1 and some other cancer related genes to disturb cancer hallmark pathways including p53 signaling pathway. It had been reported that PVT1 was associated with multiple types of human malignancies, including prostate cancer, pancreatic ductal adenocarcinoma, ovarian cancer etc [37, 53-55]. In addition, the hub IncRNA DLEU2 competitively regulated pathway up to 14 pathways. It was a critical host gene of miR-15a and miR-16-1 which inhibited tumorigenicity both in vitro and in vivo and frequently deleted in malignancy [37]. The above result suggest that these hub lncRNAs such as HCG-18, DLUE2 and PVT1 may play key roles in the initiation and progression of kidney cancer.

To further detect key lncRNAs of competitive regulation in the KIRC relevant modulatory network, we applied K-mean clustering method for survival analysis on hub lncRNAs in the network. The kidney cancer samples were divided into two groups basing on the expression quantity of the corresponding lncRNAs with $p$ value $=0.0378$ (Figure 2C), related Gene ID and sample ID of Figure 2B were in Supplementary Files (Supplementary Table S6). Meanwhile, each hub lncRNA have been applied for survival analysis separately (Supplementary Figure S4, Supplementary Table S3). Then, two groups of patients including high expression and low expression group were divided based on the mean value of the expression quantity of each hub lncRNA. The results showed that most hub lncRNA can't divide two groups very well for survival analysis separately. It suggested that these hub lncRNAs may play a coordinately regulated role in KIRC. We have applied survival analysis for TCGA data set of prostate cancer, as the same method with KIRC dataset. We applied $\mathrm{K}$-mean clustering method for survival analysis on hub lncRNAs in the PRAD regulated network. The prostate cancer samples were divided into two groups basing on the expression quantity of hub lncRNAs in the network with $p$ value $=0.0118$ (Supplementary Figure S5).
Furthermore, we performed the survival analysis for each hub lncRNA separately. Firstly, we divided the patients into two groups based on the mean value of the expression quantity of each hub lncRNA. Then, we applied survival analysis for the two groups of patients. As a result, all the hub lncRNA can't divide two groups very well for survival analysis separately (Supplementary Figure S6). It suggested that these hub lncRNAs may play a coordinately regulated role, the results showed consistency with the KIRC data set. These indicate that expression of these lncRNAs were associated with kidney cancer patient survival. Furthermore, most of hub lncRNAs exhibit high expression in the high risk group comparing with that in the low risk group (Figure 2B). The above result provided further evidence for the potential key roles of hub lncRNAs in kidney cancer.

\section{Reproducibility and robustness analyses}

\section{Reproducibility analysis}

In this section, we aim to evaluated the reproduciblity of Subpathway-LNCE, we applied Subpathway-LNCE with three independent prostate cancer data sets from three different resource, including Sequence Read Archive (SRA), TCGA Data Portal (TCGA) and Gene Expression Omnibus (GEO), details see methods. We focused on pathways that rank on top 15 of the result list from these three different data sets. Hypergeometric test was used to evaluate the significance of shared pathways between any two data sets from different resource. We found that pathways shared between SRA and TCGA with $p$ value $=5.66 \mathrm{e}-10$, and the pathways up to $80 \%$ were associated with cancers. Hypergeometric test of shared pathways between TCGA and GEO under a threshold of $p=8.56 \mathrm{e}-7$, whereas pathways shared between SRA and GEO with $p$ value $=1.84 \mathrm{e}-5$ (Figure 3). Futhermore, the number of pathways shared among SRA, GEO and TCGA data sets result was up to 6, and outstandingly, all shared pathways had been well reported associated with cancers. We further restriction to top 10 pathways in the result list (Supplementary Figure S2), the results were above all, though three independent prostate the data sets was from three different resource. We have further analyzed the results of subpathway-LNCE based on different $P$ value of differential expression gene $(0.05,0.1,0.2)$ in the same data sets for all three different data resources. And we focused on pathways that rank on top 15 of the result list from different $P$ value of differential expression gene in the same data sets for all three different data sets. The number of pathways shared under different $P$ value was up to 10 in the TCGA data set, and in the other data set the number also up to 8 and 7 , respectively (Supplementary Figure S7). In any two different $P$ value, the hypergeometric test of shared pathways was significant. All above indicated that the results obtained using Subpathway-LNCE were reproducible. 


\section{Robustness analysis}

We used two methods to test the stability of Subpathway-LNCE, namely randomly introducing noise in the matched mRNA-lncRNA expression profiles and disturbing LRSP. We firstly randomly deleted mRNAs and lncRNAs from mached mRNA-lncRNA expression profiles, and deleted percentage from $5 \%$ to $30 \%$, at $5 \%$ intervals. Then we repeated the deleted process 100 times and applied the Subpathway-LNCE method on noisily matched mRNA-lncRNA expression profiles. We calculated the mean ratio of recall significant pathways comparing to original significant pathways (FDR $<0.01$ ). In general, the deleted percentage increased, the overlap of significant pathways fell slowly. The SubpathwayLNCE shown the best stability when the deleted ratio was $5 \%$, recalling more than $90 \%$ of the pathways. Even after removal of up to $15 \%$ of the expression data, the recalling still more than $70 \%$. It indicated that Subpathway-LNCE was robust when the expression data was noise.

We next randomly deleted the edges within LRSPs from $5 \%$ to $30 \%$, at $5 \%$ intervals, then we repeated the deleted process 100 times and applied the SubpathwayLNCE method on SRA prostate data sets for each disturbed LRSP. The mean ratio of recall significant pathways was calculated comparing to original significant pathways (FDR $<0.01$ ). We found that as the deleted percentage increased, the overlap of significant pathways fell slowly (Figure 3), and the recalling still more than $60 \%$ even after disturbing up to $25 \%$ edges of LRSPs. Furthermore, Robustness analysis of the KIRC dataset was consistent with the above result (Supplementary Figure S1). Above all, it suggested that Subpathway-LNCE method was robust in resisting the disturbance of expression data and LRSPs.

\section{A Calcium signaling pathway}
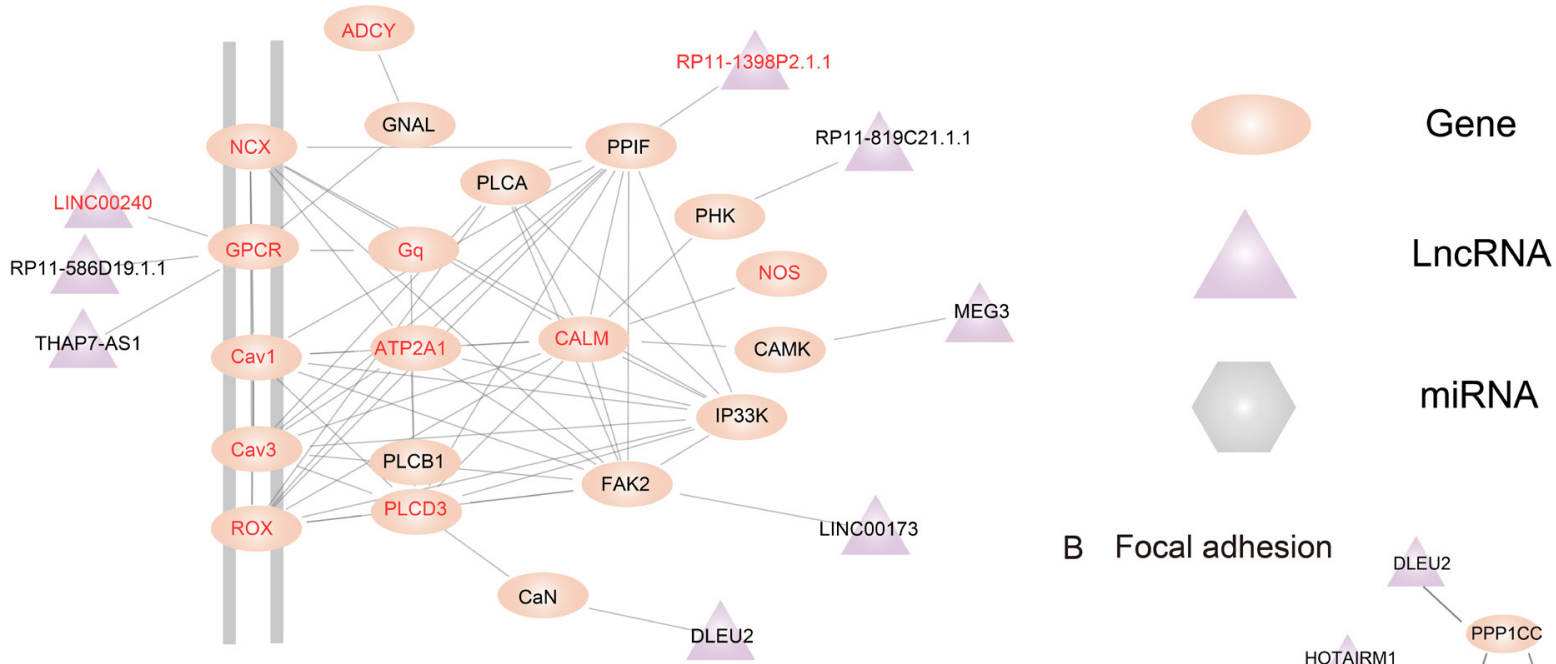

B Focal adhesion DLEU2 DLEU2

\section{Phosphatidylinositol signaling system}
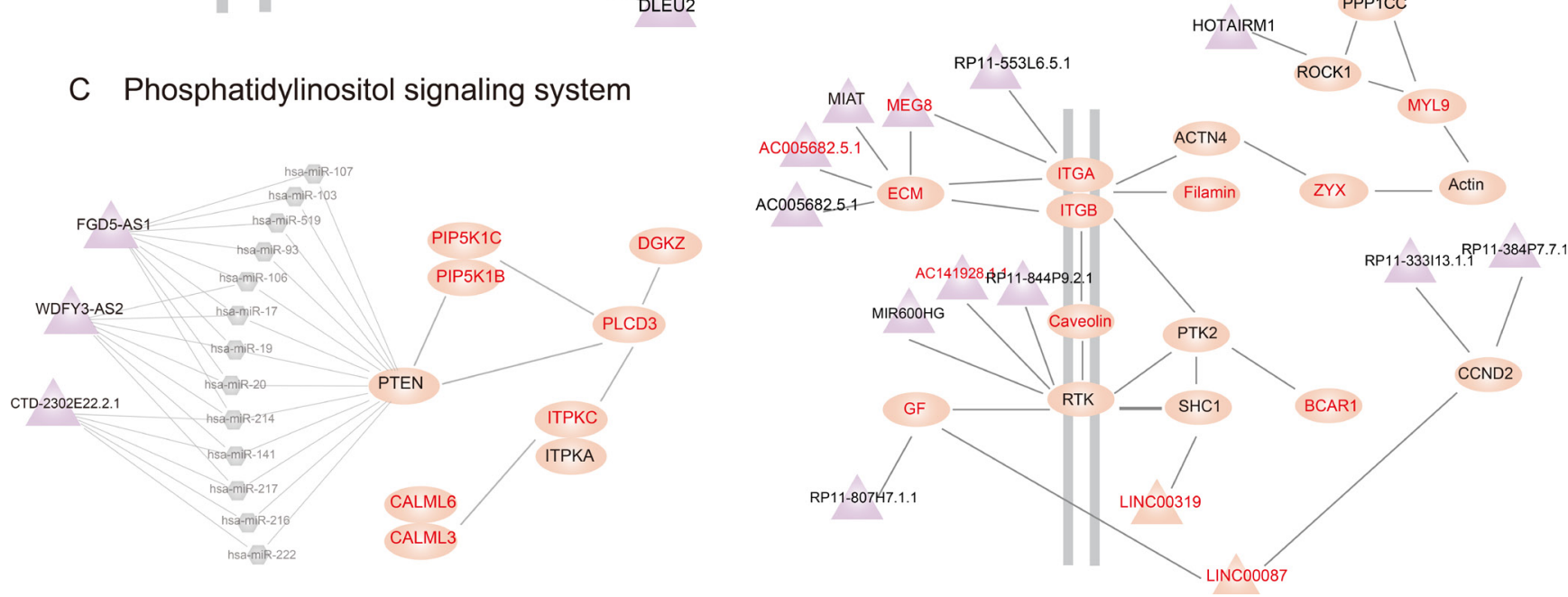

Figure 2: Subpathways identified using the subpathway-LNCE method. Ellipse, triangle and hexagon nodes represent genes, lncRNAs and miRNAs, respectively. Red and balck node labels represent differential and non-differential genes/lncRNAs, respectively, and gray node labels represent miRNAs. (A) Calcium signaling subpathway (path: 04020_1, FDR=7.87E-14). (B) Focal adhesion subpathway (path: 04070_1, FDR=7.19E-12). (C) Phosphatidylinositol signaling system subpathway (path:00030_1, FDR=0.20E-2). 


\section{DISCUSSION}

Recently, lncRNAs have been found can function as competitors of mRNAs for miRNA binding, thereby competitively regulating mRNA expression levels and maintain normal biological functions $[9,10,14]$. This regulatory mechanism may help understand biological problems and organism complexity. Disturbances of these lncRNA competitively regulated functions may led to diseases, but on the other hand, a better understanding this regulation may offer opportunities for new therapies. However, to the best of our current knowledge, there are few methods specifically designed to identify lncRNAs competitively regulated functions and the functional roles of these competitive regulation lncRNAs have not be well characterized in diseases. In this study, we proposed a novel method called Subpathway-LNCE, to identify lncRNAs competitively regulated signal subpathways underlying certain conditon, providing a powerful tool for exploring the regulation function of lncRNAs in human disease.
Subpathway-LNCE which integrated lncRNAmRNA expression profile and pathway topologies was specifically designed to identify lncRNAs competitively regulated functions. It considers several important aspects as follows. Firstly, lncRNA, which play important roles in various biological processes $[5,6]$, represent a new regulatory layer and should be included in the pathway analysis. Second, when locating candidate subregions we take advantage of pathway topologies along with lncRNAs competitively regulated genes embedded in different pathways, which was better to reflect the transmission of disease signals. LncRNAs have sponge features which can competitively regulated biological pathways and thus play critical roles in the initiation and progression of diseases such as tumor [14], it is thus necessary to integrative analyze the joint effect of genes and lncRNAs that competitively regulated them by considering pathway topologies. Third, we have adopted a strategy of subpathway, rather than completely pathways, is more subtly explainable to the etiology of diseases. Moreover, concentrating more attention on subpathways rather than entire pathways
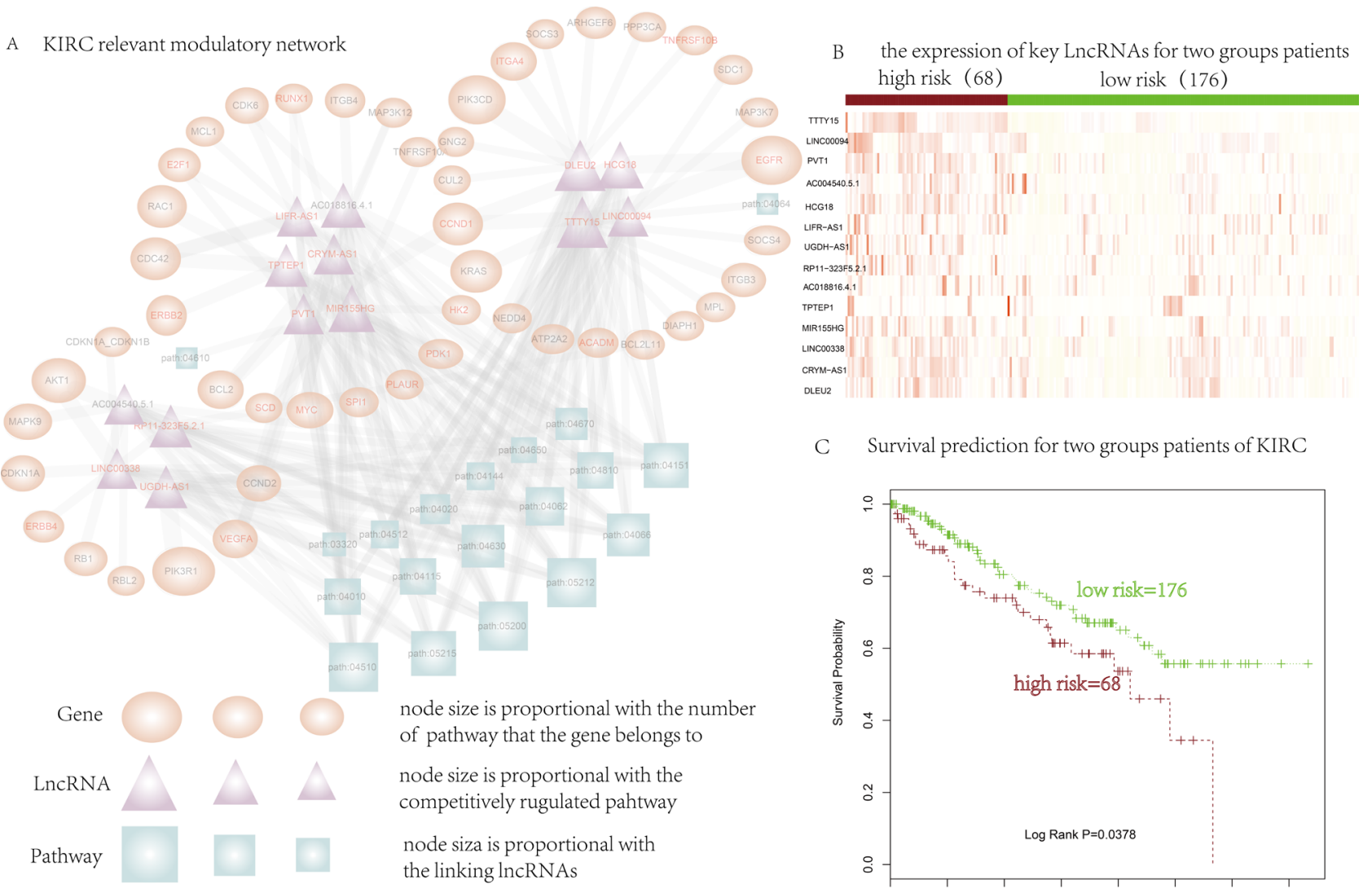

C Survival prediction for two groups patients of KIRC

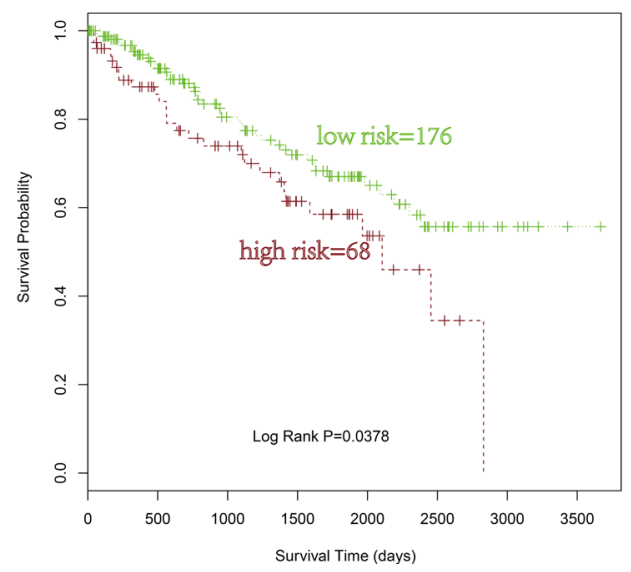

Figure 3: (A) KIRC-relevant lncRNA competitively regulated signal subpathways network. Circle and triangle nodes represent genes and lncRNAs, respectively, and square represent subpathway. The lncRNA/subpathway node size is proportional to the number of directly interacted subpathways/lncRNAs, and the gene node size is proportional to the number of appeared subpathways. The edge width of IncRNA-gene pair is proportional to the number of subpathways they involved in. (B) The expression quantity of key LncRNAs (rows) for two groups patients (colums). (C) Kaplan-Meier survival analysis of two groups of patients with different clinical outcomes. Survival days are shown along the $\mathrm{X}$ axis. Overall survival rates are shown along the $\mathrm{Y}$ axis. 
might be recall and identify more biologically meaningful pathways and dissect the functional roles of lncRNAs. The input data of Subpathway-LNCE method needs matched lncRNA-mRNA expression profiles, Subpathway-LNCE provide a flexible tool to identify lncRNA competitively regulated signal subpathways underlying certain condition, and help to expound the functional roles of lncRNAs in various status.

In the result section, we applied Subpathway-LNCE with SRA data set of prostate cancer and choose three different sub region from different aspects to elaborate the effective of our methods. The first is the significant subpathway was path:04020_1, which was a subregion of calcium signaling pathway. Down regulation of 1 ncRNA MEG3 had been reported closely associated with several cancers $[25,26]$. Moreover, MEG3 played an important role in the molecular etiology of prostate cancer, which suggested the potential application of MEG3 in prostate cancer therapy [27]. The second was path: 04510_1, an important sub region within focal adhesion pathway. We focused the upstream protein of this sub region, which were more important because it influenced other genes/ proteins downstream. Interestingly, the regulator of the upstream protein was four lncRNAs, which may play an important role for coordinated regulation and function. The third subpathway, path: 04070_1, was a part of Phosphatidylinositol signaling system. In this subpathway, we concerned the miRNAs behind PTEN and lncRNAs which was regulator of PTEN, and most miRNAs were associated with cancers. Meanwhile, We have supplied the other information of miRNA for the other two subpathways in Supplementary Files. (Supplementary Tables S4, S5). To further explore key LncRNAs of competitive regulation concerted to multiple subpathways, we constructed KIRC relevant modulatory network. In the KIRC relevant modulatory network, the modulatory relationship HCG18EGFR appeared up to 10 pathways, of which 7 pathways were cancer-related such as focal adhesion, such as MAPK signaling pathway and etc. TCGA data set include miRNA expression data, we further analyzed the expression relationship between IncRNA, miRNA and mRNA. The miR-146a-5p was the micRNA shared by HCG18EGFR, we found that the expression relationship between both HCG18-EGFR and miR-146a-5p was significantly negative. Moverer, miR-146a-5p have differential effects on growth and metastatization on cancer [56]. The differential hub lncRNA PVT1 was also competitively regulated CCND1 and some other cancer related genes to disturb cancer hallmark pathways including p53 signaling pathway. It had been reported that PVT1 was associated with multiple types of human malignancies, including prostate cancer, pancreatic ductal adenocarcinoma, ovarian cancer etc [53-55]. We found that PVT1 was the target of miR-106a-5p and miR-20b-5p, and the expression relationship between PVT1 and both miRNAs were significantly negative. Then we applied K-mean clustering method for survival analysis on hub lncRNAs in the network to detected key LncRNAs of competitive regulation in the KIRC relevant modulatory network. The kidney cancer samples were divided into two groups based on the expression value of the corresponding lncRNAs with $\log$ rank $p$ value $=0.0378$. It suggested LncRNA may be responsible for explaining disease processes thereby presenting opportunities for new therapies.

In this study, there are differences among three data sets, including experimental method, operational approach etc. So we considered false discovery rate (FDR) to identify differentially expressed genes in different data sets. Actually, many researchers took different threshold values among different data sets for identifying differential genes. For example, Liang et al. applied different FDRs in their study [57]. We then further explored the fold change of these differential genes in each dataset, the mean value of fold change among three different data sets all $>=1.5$ (Supplementary Figure S3). We used Pearson Correlation Coefficient to evaluate co-expression for any pair of relations in the candidate lncRNA-mRNA network based on matched lncRNA and mRNA expression profiles. And there are some convenient tools can evaluate coexpression. However, any other suitable method, such as Weighted Gene Co-Expression Network Analysis [58], can be used to calculate this co-expression coefficient.

In order to ensure the reliability of data, the mRNA-miRNA interactions were experimentally validated, collected from TarBase [59], mirTarBase [60], mir2Disease [61], miRecords (V4.0) [62]. The lncRNAmiRNA interactions were predicted using TargetScan (v.6.0) [63], PITA (March 2007 version) [64] , miRanda (Nov. 2010 version) [65] and RNAhybrid (v.2.1.1) [66] with default parameters. Then we have integrated the AGO-CLIP-seq data set into the pipeline to identify miRNA-binding sites on lncRNA sequences which have experimentally supported. By integrating genome coordinates of CLIP-seq peaks and predicted miRNAbinding sites, the reliability of data was further ensured. We have used published scientific documentation confirming the results from Subpathway-LNCE. For example, in the KIRC relevant modulatory network, the differential hub lncRNA PVT1 was also competitively regulated CCND1 and some other cancer related genes to disturb cancer hallmark pathways including p53 signaling pathway. It had been reported that PVT1 was associated with multiple types of human malignancies, including prostate cancer, pancreatic ductal adenocarcinoma, ovarian cancer etc [53-55]. The hub lncRNA DLEU2 was a critical host gene of miR-15a and miR-16-1 which inhibited tumorigenicity both in vitro and in vivo and frequently deleted in malignancy [37]. Although we didn't use experiments to confirm the results, we have supplied specific binding sites about lncRNA-miRNA data to guide a further experiment to verify the predicted results for researchers (Supplementary Table S7). 
In summary, Subpathway-LNCE, a novel method that designed to identify lncRNAs competitively regulated signal subpathways, can not only help to understanding the molecular mechanism of diseases, but also will lead to important insight into the functional roles of lncRNAs in pathological states. Moreover, we have implemented Subpathway-LNCE method as an R-based package, which is publicly available on https://cran.rstudio.com/ web/packages/SubpathwayLNCE/, and our tool provided a flexible usage for changing the significant positive threshold of $r$ value.

\section{MATERIALS AND METHODS}

\section{Materials}

\section{Prostate cancer datasets}

We analyzed three independent prostate data sets, the three prostate data sets was obtained from different data sources: Sequence Read Archive (SRA, SRP002628) [21], The Cancer Genome Atlas dataset (TCGA, https:// tcga-data.nci.nih.gov/tcga/) and Gene Expression Omnibus (GEO, GSE23316) [67], and each of them included cancer samples and normal samples.

(i) SRA data set of prostate cancer

To obtain transcription level of lncRNAs and mRNAs, firstly we used TopHat mapping reads to the reference genome (hg19) [68], next we estimated the relative abundances of each transcript using Cufflinks with transcript annotation file [69]. Thus, we obtained RPKM lncRNA and mRNA expression profiles. Whereas, to get read counts expression profiles, RNA-Seq reads were mapped to the reference genome (hg19) using Tophat [68], then expression level was extracted using easyRNAseq method [70]. We used DESeq method to identify differentially expressed mRNAs based on read count expression profies [71]. The matched lncRNA and mRNA expression profiles, which included 10 normal samples and 20 cancer samples. MRNAs and lncRNAs were considered differentially expressed under a threshold of FDR $=0.1$.

(ii) TCGA data set of prostate cancer

The PRAD data set was download from TCGA, and corresponding clinical information was also obtained. The matched mRNA/lncRNA expression profile for prostate cancer were extracted according to our previous study [72]. In brief, RNA-seqV2 data were downloaded from TCGA level 3 data sets, and RPKM (Reads Per Kilobase per Million mapped reads) values for lncRNA/ mRNA were recalculated using the following method: $\mathrm{RPKM}=\left(\right.$ raw read count $\left.\times 10^{\wedge} 9\right) /($ total reads $)$, where raw read counts $=$ sum of raw read counts in all exons mapped entirely within the lncRNA/mRNA locus; total reads $=$ sum of raw read counts calculated for all exons of a single sample; = sum of length of exons mapped the LncRNA / mRNA locus. Annotation of exons mapping to
lncRNA/mRNA was extracted from GENCODE (V19). Finally, the matched lncRNA and mRNA expression profiles included 494 cancer samples and 42 normal samples. We used DESeq method to identify differentially expressed mRNAs based on read count expression profiles [71]. A mRNA was considered to be differentially expressed with false discovery rate $(\mathrm{FDR})<1 \mathrm{e}-4$.

(iii) GEO data set of prostate cancer

We mapped 604258 probe sequences (25 bp in length) to annotated files from GENCODE (GRCH37) with BLAST under probe re-annotation pipeline (see Supplementary Files), resulted with 17254 mRNAs and 3495 lncRNAs. We obtained matched lncRNA and mRNA expression profiles, which included 6 normal samples and 12 cancer samples. MRNAs were considered differentially expressed under a threshold of FDR $=0.1$ of Student's $t$ test.

\section{KIRC datasets}

The KIRC data set was download from TCGA, and corresponding clinical information was also obtained. Details of these data sets were processed similar with TCGA data set of prostate cancer, which has described above. And the matched lncRNA and mRNA expression profiles included 71 normal samples and 255 cancer samples.

\section{LUAD datasets}

The LUAD data set was download from TCGA. Details of the data-set were processed similar with TCGA data set of prostate cancer, which has described above. And the matched lncRNA and mRNA expression profiles included 52 normal samples and 494 cancer samples.

\section{Methods}

Subpathway-LNCE was developed to identify lncRNA competitively regulated signal subpathways underlying certain conditon. The schematic overview is shown in Figure 4. It includes four main parts: (i) Firstly, we constructed candidate lncRNA-mRNA competitively regulated network; (ii) KEGG signal pathways were converted into undirected graphs with genes as nodes and regulated relations as edges, we reconstructed conditionspecific lncRNA competitively regulated signal pathways (LRSP) based on matched lncRNA and mRNA expression profiles and IncRNA-mRNA competitively regulated network; (iii) We mapped competing lncRNAs and interesting genes into LRSP, then locates subpahtways within pathways according to the "lenient distance" similarity method; (iv) We evaluated the significance of candidate subpathways by using the Wallenius approximation. The details are displayed below. 


\section{Constructing candidate IncRNA-mRNA interaction}

Firstly, we collected lncRNA-associated competing triplets (lncRNA-miRNA-mRNA relationships). The IncRNA-miRNA interactions were obtainted from our previously work and StarBase, whereas experimentally validated mRNA-miRNA interactions were collected from TarBase [59], mirTarBase [60], mir2Disease [61], miRecords (V4.0) [62]. Then, we constructed candidate LncRNA-mRNA competitively regulated relationships based on their shared miRNAs. For each lncRNA, we identify its candidate competing mRNAs as follows: (i) hypergeometric test of shared miRNAs under a threshold of $p=0.05$ (ii) Jaccard Coefficient of shared miRNAs rank at top $20 \%$. In order to ensure the reliability of data, those relationships which satisfied with both criteria were retained. Finally, we obtained candidate LncRNA-mRNA competitively regulated network included 6722 lncRNAmRNA interactions among 1527 genes and 798 LncRNAs.

\section{Reconstructing condition-specific LncRNA- regulated signal pathways}

\section{Linking LncRNAs to regulated-mRNAs within pathway graphs}

We converted 191 KEGG signal pathways into undirected graphs kept original pathway structural information using our previously developed $\mathrm{R}$ packages [19]. We used Pearson Correlation Coefficient to evaluate co-expression for any pair of relations in the candidate IncRNA-mRNA network based on matched lncRNA and mRNA expression profiles, those $r$ value had reached a significant positive threshold were retained $(p<0.05)$ based on Fisher's Z transform [73]. Then, these lncRNAs were embedded into pathway graphs as nodes by linking to their regulated-mRNAs. Finally, we obtained conditionspecific lncRNA competitively regulated signal pathways (LRSP), which included lncRNA nodes and IncRNAmRNA competitively regulated edges.

\section{Locate subpathways competing regulated by IncRNAs}

LncRNAs involved in the competing regulation and genes of interests were regarded as signature nodes. These nodes combined with topology of LRSP can help us efficiently positioning lncRNA-regulated subregions. We first mapped signatures nodes into LRSP, then locating subpathways competing regulated by lncRNAs used "lenient distance" similarity combined with network topology structure. In brief, we calculated the shortest path between any two signature nodes, if the number of molecules between each signature pairs was no longer than n, then they were merged into one nodes. Finally, the number of nodes in the molecule sets within pathway

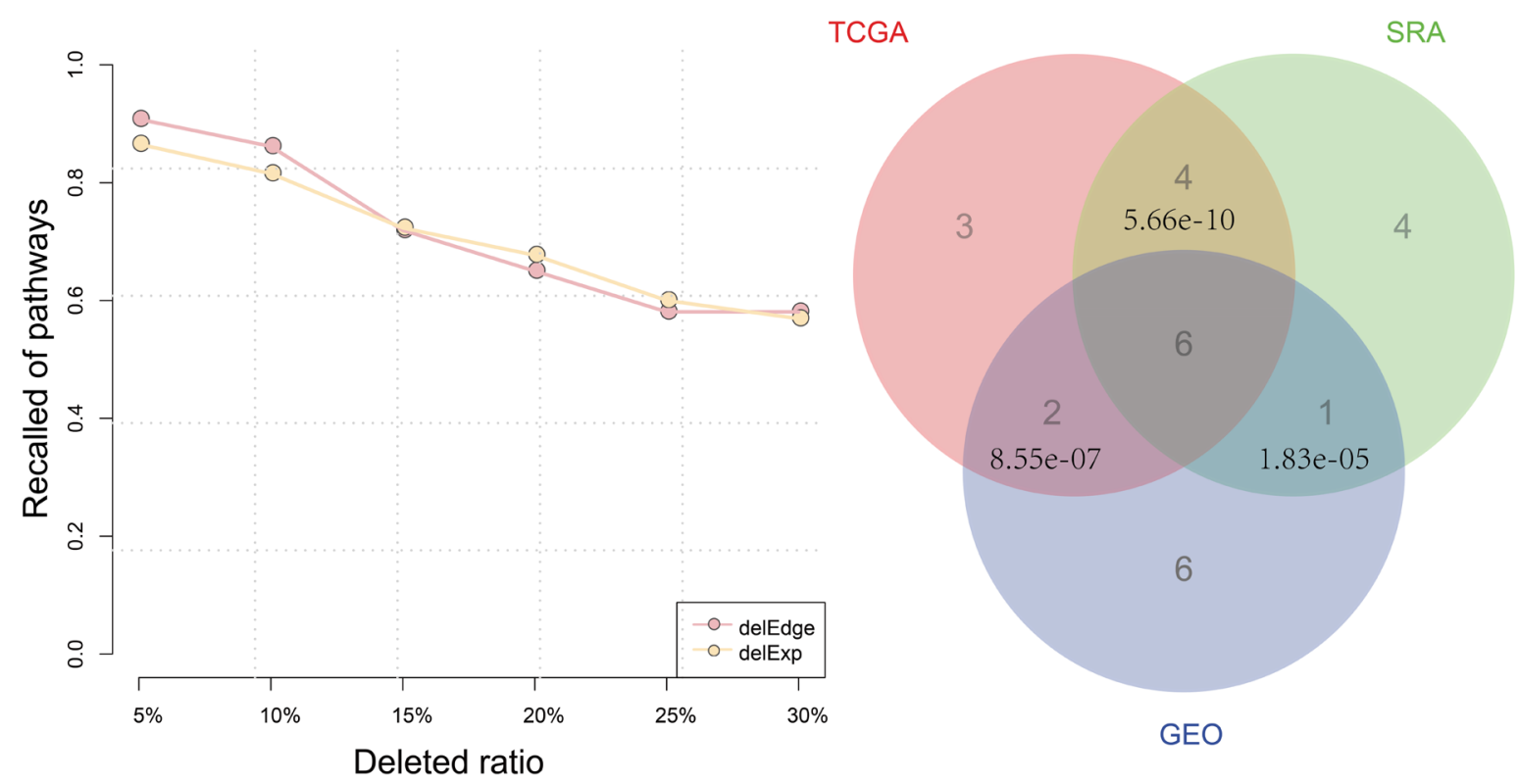

Figure 4: Reproducibility and robustness analyses. (A) Robustness analysis. Pink line shows the mean ratio of recalled pathways using Subpathway-LNCE method for prostate cancer data set after randomly deleting $N \%$ of genes and miRNAs from the corresponding profiles, where $N=5,10, \ldots, 30$. Light green line shows the mean ratio of for prostate cancer data set recalled pathways using SubpathwayLNCE after randomly deleting $N \%$ of the edges in each RMPG, where $N=5,10, \ldots, 30$. (B) Reproducibility analysis. Venn diagram depicts top 15 pathways identified by Subpathway-LNCE in three independent prostate cancer data sets from three different resource, including Sequence Read Archive (SRA), TCGA Data Portal (TCGA) and Gene Expression Omnibus (GEO). 
no less than s were regarded as candidate subpahtways competing regulated by LncRNAs. The $n$ and $s$ parameters control the intensity of regulated signals and the size of candidate subpathways, respectively. We used $n=1$ and $s=8$ as default parameters.

\section{Evaluated the significance of candidate subathways}

To estimate whether the candidate subpathways were competing regulated by lncRNAs comparing random, we used the Wallenius approximation methods to estimate the significance of candidate subpathways. The following parameters were needed: (i) the number of interesting mRNAs (x); (ii) the number of background mRNAs (n); (iii) the number of background mRNAs involved in this subpathways (m1); (iv) the number of interesting mRNAs annotated into this subpathway (m2); (v) the weight of this subpathway (w), which suggested the intensity of competing regulation by lncRNAs involved in this subpathways. The weight of the subpathway was computed as follows:

In above formula, parameter $\mathrm{P}_{\mathrm{G}}$ is the number of mRNAs of this subpathways, and parameter $G_{L}$ is the number of mRNAs competitively regulated by lncRNAs within this subpathway. $\beta$ is parameter of control (In this study $\beta=1$ ). The Wallenius approximation methods was executed using R package BiasedUrn [20].

$$
W=1+\beta\left(-\log _{2}\left(\frac{G_{L}}{P_{G}}\right)\right)
$$

\section{Survival analysis}

K-mean clustering was used to classify KIRC (PRAD) patients into two groups by expression of hub IncRNAs in the IncRNA competitively regulated subpathway network. Then, the Kaplan-Meier method was used to evaluate the difference of survival time between these two groups, and statistical significance was estimated by log-rank test.

\section{CONFLICTS OF INTEREST}

There have no competing interests.

\section{FUNDING}

This work was supported in part by the National High Technology Research and Development Program of China [863 Program, Grant Nos. 2014AA021102], the National Program on Key Basic Research Project
[973 Program, Grant Nos. 2014CB910504], the National Natural Science Foundation of China [Grant Nos. 91439117, 61473106, 81572341 and 31401127], China Postdoctoral Science Foundation [2016M591544], Harbin medical university scientific research innovation fund [2016JCZX48].

\section{Data availability}

All data underlying the findings are fully available without restriction. All relevant data are within the paper and its Supporting Information files.

\section{REFERENCES}

1. Guttman M, Amit I, Garber M, French C, Lin MF, Feldser D, Huarte M, Zuk O, Carey BW, Cassady JP, Cabili MN, Jaenisch R, Mikkelsen TS, et al. Chromatin signature reveals over a thousand highly conserved large non-coding RNAs in mammals. Nature. 2009; 458:223-227.

2. Iyer MK, Niknafs YS, Malik R, Singhal U, Sahu A, Hosono Y, Barrette TR, Prensner JR, Evans JR, Zhao S, Poliakov A, Cao X, Dhanasekaran SM, et al. The landscape of long noncoding RNAs in the human transcriptome. Nat Genet. 2015; 47:199-208.

3. Wang KC, Chang HY. Molecular mechanisms of long noncoding RNAs. Mol Cell. 2011; 43:904-914.

4. Wilusz JE, Sunwoo H, Spector DL. Long noncoding RNAs: functional surprises from the RNA world. Genes Dev. 2009; 23:1494-1504.

5. Ponting CP, Oliver PL, Reik W. Evolution and functions of long noncoding RNAs. Cell. 2009; 136:629-641.

6. Mercer TR, Dinger ME, Mattick JS. Long non-coding RNAs: insights into functions. Nat Rev Genet. 2009; 10: 155-159.

7. Liu B, Sun L, Liu Q, Gong C, Yao Y, Lv X, Lin L, Yao H, Su F, Li D, Zeng M, Song E. A cytoplasmic NFkappaB interacting long noncoding RNA blocks IkappaB phosphorylation and suppresses breast cancer metastasis. Cancer Cell. 2015; 27:370-381.

8. Barsyte-Lovejoy D, Lau SK, Boutros PC, Khosravi F, Jurisica I, Andrulis IL, Tsao MS, Penn LZ. The c-Myc oncogene directly induces the H19 noncoding RNA by allele-specific binding to potentiate tumorigenesis. Cancer Res. 2006; 66:5330-5337.

9. Ebert MS, Sharp PA. Emerging roles for natural microRNA sponges. Curr Biol. 2010; 20:R858-861.

10. Tay Y, Rinn J, Pandolfi PP. The multilayered complexity of ceRNA crosstalk and competition. Nature. 2014; 505:344-352.

11. Karreth FA, Pandolfi PP. ceRNA cross-talk in cancer: when ce-bling rivalries go awry. Cancer Discov. 2013; 3:1113-1121.

12. Poliseno L, Salmena L, Zhang J, Carver B, Haveman WJ, Pandolfi PP. A coding-independent function of gene and 
pseudogene mRNAs regulates tumour biology. Nature. 2010; 465:1033-1038.

13. Wang Y, Xu Z, Jiang J, Xu C, Kang J, Xiao L, Wu M, Xiong J, Guo X, Liu H. Endogenous miRNA sponge lincRNA-RoR regulates Oct4, Nanog, and Sox2 in human embryonic stem cell self-renewal. Dev Cell. 2013; 25:69-80.

14. Liang WC, Fu WM, Wong CW, Wang Y, Wang WM, Hu GX, Zhang L, Xiao LJ, Wan DC, Zhang JF, Waye MM. The lncRNA H19 promotes epithelial to mesenchymal transition by functioning as miRNA sponges in colorectal cancer. Oncotarget. 2015; 6:22513-22525. doi: 10.18632/ oncotarget.4154.

15. Sumazin P, Yang X, Chiu HS, Chung WJ, Iyer A, LlobetNavas D, Rajbhandari P, Bansal M, Guarnieri P, Silva J, Califano A. An extensive microRNA-mediated network of RNA-RNA interactions regulates established oncogenic pathways in glioblastoma. Cell. 2011; 147:370-381.

16. Liu K, Yan Z, Li Y, Sun Z. Linc2GO: a human LincRNA function annotation resource based on ceRNA hypothesis. Bioinformatics. 2013; 29:2221-2222.

17. Jiang Q, Ma R, Wang J, Wu X, Jin S, Peng J, Tan R, Zhang T, Li Y, Wang Y. LncRNA2Function: a comprehensive resource for functional investigation of human lncRNAs based on RNA-seq data. BMC Genomics. 2015; 16:S2.

18. Li C, Li X, Miao Y, Wang Q, Jiang W, Xu C, Li J, Han J, Zhang F, Gong B, Xu L. SubpathwayMiner: a software package for flexible identification of pathways. Nucleic Acids Res. 2009; 37:e131.

19. Li C, Han J, Yao Q, Zou C, Xu Y, Zhang C, Shang D, Zhou L, Zou C, Sun Z, Li J, Zhang Y, Yang H, et al. Subpathway-GM: identification of metabolic subpathways via joint power of interesting genes and metabolites and their topologies within pathways. Nucleic Acids Res. 2013; 41:e101.

20. Epstein MP, Duncan R, Jiang Y, Conneely KN, Allen AS, Satten GA. A permutation procedure to correct for confounders in case-control studies, including tests of rare variation. Am J Hum Genet. 2012; 91:215-223.

21. Kannan K, Wang L, Wang J, Ittmann MM, Li W, Yen L. Recurrent chimeric RNAs enriched in human prostate cancer identified by deep sequencing. Proc Natl Acad Sci USA. 2011; 108:9172-9177.

22. Berridge MJ, Lipp P, Bootman MD. The versatility and universality of calcium signalling. Nat Rev Mol Cell Biol. 2000; 1:11-21.

23. Tavecchio M, Lisanti S, Lam A, Ghosh JC, Martin NM, O'Connell M, Weeraratna AT, Kossenkov AV, Showe LC, Altieri DC. Cyclophilin D extramitochondrial signaling controls cell cycle progression and chemokine-directed cell motility. J Biol Chem. 2013; 288:5553-5561.

24. Zhang J, Yao T, Wang Y, Yu J, Liu Y, Lin Z. Long noncoding RNA MEG3 is downregulated in cervical cancer and affects cell proliferation and apoptosis by regulating miR-21. Cancer Biol Ther. 2015:0.

25. Peng W, Si S, Zhang Q, Li C, Zhao F, Wang F, Yu J, Ma R. Long non-coding RNA MEG3 functions as a competing endogenous RNA to regulate gastric cancer progression. J Exp Clin Cancer Res. 2015; 34:79.

26. Xia Y, He Z, Liu B, Wang P, Chen Y. Downregulation of Meg3 enhances cisplatin resistance of lung cancer cells through activation of the WNT/beta-catenin signaling pathway. Mol Med Rep. 2015; 12:4530-4537.

27. Luo G, Wang M, Wu X, Tao D, Xiao X, Wang L, Min F, Zeng F, Jiang G. Long Non-Coding RNA MEG3 Inhibits Cell Proliferation and Induces Apoptosis in Prostate Cancer. Cell Physiol Biochem. 2015; 37:2209-2220.

28. Insua-Rodriguez J, Oskarsson T. The extracellular matrix in breast cancer. Adv Drug Deliv Rev. 2015.

29. Alfano M, Canducci F, Nebuloni M, Clementi M, Montorsi F, Salonia A. The interplay of extracellular matrix and microbiome in urothelial bladder cancer. Nat Rev Urol. 2015.

30. de Brot S, Ntekim A, Cardenas R, James V, Allegrucci C, Heery DM, Bates DO, Odum N, Persson JL, Mongan NP. Regulation of vascular endothelial growth factor in prostate cancer. Endocr Relat Cancer. 2015; 22:R107-123.

31. Liu ZQ, Fang JM, Xiao YY, Zhao Y, Cui R, Hu F, Xu Q. Prognostic role of vascular endothelial growth factor in prostate cancer: a systematic review and meta-analysis. Int J Clin Exp Med. 2015; 8:2289-2298.

32. Kobayashi T, Nakamura E, Shimizu Y, Terada N, Maeno A, Kobori G, Kamba T, Kamoto T, Ogawa O, Inoue T. Restoration of cyclin D2 has an inhibitory potential on the proliferation of LNCaP cells. Biochem Biophys Res Commun. 2009; 387:196-201.

33. Vasiljevic N, Ahmad AS, Thorat MA, Fisher G, Berney DM, Moller H, Foster CS, Cuzick J, Lorincz AT. DNA methylation gene-based models indicating independent poor outcome in prostate cancer. BMC Cancer. 2014; 14:655.

34. Zhang X, Weissman SM, Newburger PE. Long intergenic non-coding RNA HOTAIRM1 regulates cell cycle progression during myeloid maturation in NB4 human promyelocytic leukemia cells. RNA Biol. 2014; 11:777-787.

35. Diaz-Beya M, Brunet S, Nomdedeu J, Pratcorona M, Cordeiro A, Gallardo D, Escoda L, Tormo M, Heras I, Ribera JM, Duarte R, de Llano MP, Bargay J, et al. The lincRNA HOTAIRM1, located in the HOXA genomic region, is expressed in acute myeloid leukemia, impacts prognosis in patients in the intermediate-risk cytogenetic category, and is associated with a distinctive microRNA signature. Oncotarget. 2015; 6:31613-31627. doi: 10.18632/oncotarget.5148.

36. Kasar S, Underbayev C, Yuan Y, Hanlon M, Aly S, Khan H, Chang V, Batish M, Gavrilova T, Badiane F, Degheidy H, Marti G, Raveche E. Therapeutic implications of activation of the host gene (Dleu2) promoter for miR-15a/16-1 in chronic lymphocytic leukemia. Oncogene. 2014; 33:3307-3315.

37. Lerner M, Harada M, Loven J, Castro J, Davis Z, Oscier D, Henriksson M, Sangfelt O, Grander D, Corcoran MM. DLEU2, frequently deleted in malignancy, functions as a critical host gene of the cell cycle inhibitory microRNAs miR-15a and miR-16-1. Exp Cell Res. 2009; 315:2941-2952. 
38. Kandoth C, McLellan MD, Vandin F, Ye K, Niu B, Lu C, Xie M, Zhang Q, McMichael JF, Wyczalkowski MA, Leiserson MD, Miller CA, Welch JS, et al. Mutational landscape and significance across 12 major cancer types. Nature. 2013; 502:333-339.

39. Huang YQ, Han ZD, Liang YX, Lin ZY, Ling XH, Fu X, Cai C, Bi XC, Dai QS, Chen JH, He HC, Chen YR, Jiang FN, et al. Decreased expression of myosin light chain MYL9 in stroma predicts malignant progression and poor biochemical recurrence-free survival in prostate cancer. Med Oncol. 2014; 31:820.

40. Bilir B, Osunkoya AO, Wiles WGt, Sannigrahi S, Lefebvre V, Metzger D, Spyropoulos DD, Martin WD, Moreno CS. SOX4 is essential for prostate tumorigenesis initiated by PTEN ablation. Cancer Res. 2015.

41. Milella M, Falcone I, Conciatori F, Cesta Incani U, Del Curatolo A, Inzerilli N, Nuzzo CM, Vaccaro V, Vari S, Cognetti F, Ciuffreda L. PTEN: Multiple Functions in Human Malignant Tumors. Front Oncol. 2015; 5:24.

42. Mulholland DJ, Dedhar S, Wu H, Nelson CC. PTEN and GSK3beta: key regulators of progression to androgenindependent prostate cancer. Oncogene. 2006; 25:329-337.

43. Morgan TM, Koreckij TD, Corey E. Targeted therapy for advanced prostate cancer: inhibition of the PI3K/Akt/mTOR pathway. Curr Cancer Drug Targets. 2009; 9:237-249.

44. Srivastava A, Goldberger H, Dimtchev A, Ramalinga M, Chijioke J, Marian C, Oermann EK, Uhm S, Kim JS, Chen LN, Li X, Berry DL, Kallakury BV, et al. MicroRNA profiling in prostate cancer-the diagnostic potential of urinary miR-205 and miR-214. PLoS One. 2013; 8:e76994.

45. Dhar S, Kumar A, Rimando AM, Zhang X, Levenson AS. Resveratrol and pterostilbene epigenetically restore PTEN expression by targeting oncomiRs of the miR-17 family in prostate cancer. Oncotarget. 2015; 6:27214-27226. doi: 10.18632/oncotarget.4877.

46. Maia LB, Breginski FS, Cavalcanti TC, de Souza RL, Roxo VM, Ribeiro EM. No difference in CCND1 gene expression between breast cancer patients with and without lymph node metastasis in a Southern Brazilian sample. Clin Exp Med. 2015.

47. Marampon F, Gravina GL, Ju X, Vetuschi A, Sferra R, Casimiro MC, Pompili S, Festuccia C, Colapietro A, Gaudio E, Di Cesare E, Tombolini V, Pestell RG. Cyclin D1 silencing suppresses tumorigenicity, impairs DNA double strand break repair and thus radiosensitizes androgenindependent prostate cancer cells to DNA damage. Oncotarget. 2016; 7:5383-400. doi: 10.18632/oncotarget.6579.

48. Sun CC, Li SJ, Li DJ. Hsa-miR-134 suppresses non-small cell lung cancer (NSCLC) development through downregulation of CCND1. Oncotarget. 2016; doi: 10.18632/ oncotarget.8482.

49. Melenhorst WB, Mulder GM, Xi Q, Hoenderop JG, Kimura K, Eguchi S, van Goor H. Epidermal growth factor receptor signaling in the kidney: key roles in physiology and disease. Hypertension. 2008; 52:987-993.
50. Bordi P, Del Re M, Danesi R, Tiseo M. Circulating DNA in diagnosis and monitoring EGFR gene mutations in advanced non-small cell lung cancer. Transl Lung Cancer Res. 2015; 4:584-597.

51. Tsai PC, Fu YS, Chang LS, Lin SR. Taiwan cobra cardiotoxin III suppresses EGF/EGFR-mediated epithelialto-mesenchymal transition and invasion of human breast cancer MDA-MB-231 cells. Toxicon. 2016.

52. Jet Aw S, Hong Kuick C, Hwee Yong M, Wen Quan Lian D, Wang S, Liang Loh AH, Ling S, Lian Peh G, Yen Soh S, Pheng Loh AH, Hoon Tan P, Tou En Chang K. Novel Karyotypes and Cyclin D1 Immunoreactivity in Clear Cell Sarcoma of the Kidney. Pediatr Dev Pathol. 2015; 18:297-304.

53. Ilboudo A, Chouhan J, McNeil BK, Osborne JR, Ogunwobi OO. PVT1 Exon 9: A Potential Biomarker of Aggressive Prostate Cancer? Int J Environ Res Public Health. 2015; 13.

54. Cui D, Yu CH, Liu M, Xia QQ, Zhang YF, Jiang WL. Long non-coding RNA PVT1 as a novel biomarker for diagnosis and prognosis of non-small cell lung cancer. Tumour Biol. 2015.

55. Liu E, Liu Z, Zhou Y. Carboplatin-docetaxel-induced activity against ovarian cancer is dependent on up-regulated lncRNA PVT1. Int J Clin Exp Pathol. 2015; 8:3803-3810.

56. Raimo M, Orso F, Grassi E, Cimino D, Penna E, De Pitta C, Stadler MB, Primo L, Calautti E, Quaglino P, Provero P, Taverna D. miR-146a Exerts Differential Effects on Melanoma Growth and Metastatization. Mol Cancer Res. 2016.

57. Yang Y, Han L, Yuan Y, Li J, Hei N, Liang H. Gene coexpression network analysis reveals common system-level properties of prognostic genes across cancer types. Nat Commun. 2014; 5:3231.

58. Langfelder $\mathrm{P}$, Horvath S. WGCNA: an R package for weighted correlation network analysis. BMC Bioinformatics. 2008; 9:559.

59. Vergoulis T, Vlachos IS, Alexiou P, Georgakilas G, Maragkakis M, Reczko M, Gerangelos S, Koziris N, Dalamagas T, Hatzigeorgiou AG. TarBase 6.0: capturing the exponential growth of miRNA targets with experimental support. Nucleic Acids Res. 2012; 40:D222-229.

60. Hsu SD, Lin FM, Wu WY, Liang C, Huang WC, Chan WL, Tsai WT, Chen GZ, Lee CJ, Chiu CM, Chien CH, Wu MC, Huang CY, et al. miRTarBase: a database curates experimentally validated microRNA-target interactions. Nucleic Acids Res. 2011; 39:D163-169.

61. Jiang Q, Wang Y, Hao Y, Juan L, Teng M, Zhang X, Li M, Wang G, Liu Y. miR2Disease: a manually curated database for microRNA deregulation in human disease. Nucleic Acids Res. 2009; 37:D98-104.

62. Xiao F, Zuo Z, Cai G, Kang S, Gao X, Li T. miRecords: an integrated resource for microRNA-target interactions. Nucleic Acids Res. 2009; 37:D105-110. 
63. Friedman RC, Farh KK, Burge CB, Bartel DP. Most mammalian mRNAs are conserved targets of microRNAs. Genome Res. 2009; 19:92-105.

64. Kertesz M, Iovino N, Unnerstall U, Gaul U, Segal E. The role of site accessibility in microRNA target recognition. Nat Genet. 2007; 39:1278-1284.

65. Betel D, Wilson M, Gabow A, Marks DS, Sander C. The microRNA.org resource: targets and expression. Nucleic Acids Res. 2008; 36:D149-153.

66. Rehmsmeier M, Steffen P, Hochsmann M, Giegerich R. Fast and effective prediction of microRNA/target duplexes. RNA. 2004; 10:1507-1517.

67. Consortium EP. An integrated encyclopedia of DNA elements in the human genome. Nature. 2012; 489:57-74.

68. Kim D, Pertea G, Trapnell C, Pimentel H, Kelley R, Salzberg SL. TopHat2: accurate alignment of transcriptomes in the presence of insertions, deletions and gene fusions. Genome Biol. 2013; 14:R36.
69. Trapnell C, Roberts A, Goff L, Pertea G, Kim D, Kelley DR, Pimentel H, Salzberg SL, Rinn JL, Pachter L. Differential gene and transcript expression analysis of RNA-seq experiments with TopHat and Cufflinks. Nat Protoc. 2012; 7:562-578.

70. Delhomme N, Padioleau I, Furlong EE, Steinmetz LM. easyRNASeq: a bioconductor package for processing RNASeq data. Bioinformatics. 2012; 28:2532-2533.

71. Anders S, Huber W. Differential expression analysis for sequence count data. Genome Biol. 2010; 11:R106.

72. Wang P, Ning S, Zhang Y, Li R, Ye J, Zhao Z, Zhi H, Wang T, Guo Z, Li X. Identification of lncRNA-associated competing triplets reveals global patterns and prognostic markers for cancer. Nucleic Acids Res. 2015; 43:3478-3489.

73. Best D, Roberts D. Algorithm AS 89: the upper tail probabilities of Spearman's rho. Journal of the Royal Statistical Society Series C (Applied Statistics). 1975; 24: 377-379. 